\title{
The Effects of Walking or Walking-with-Poles Training on Tissue Oxygenation in Patients with Peripheral Arterial Disease
}

\author{
Eileen G. Collins, ${ }^{1,2}$ Conor McBurney, ${ }^{3}$ Jolene Butler, ${ }^{3}$ Christine Jelinek, ${ }^{3}$ Susan O'Connell, ${ }^{3}$ \\ Cynthia Fritschi, ${ }^{2}$ and Domenic Reda ${ }^{4}$
}

${ }^{1}$ Department of Veterans Affairs, Center for Management of Complex Chronic Care, Edward Hines Jr., VA Hospital, Hines,
IL 60141, USA
${ }^{2}$ Department of Biobehavioral Health Science, College of Nursing, University of Illinois at Chicago, Chicago, IL 60612, USA
${ }^{3}$ Department of Veterans Affairs, Research \& Development, Edward Hines Jr., VA Hospital, Hines, IL 60141, USA
${ }^{4}$ Cooperative Studies Program, Department of Veterans Affairs, Edward Hines Jr., VA Hospital, Hines, IL 60141, USA

Correspondence should be addressed to Eileen G. Collins, ecollins@uic.edu

Received 3 July 2012; Revised 27 August 2012; Accepted 28 August 2012

Academic Editor: Erich Minar

Copyright (C) 2012 Eileen G. Collins et al. This is an open access article distributed under the Creative Commons Attribution License, which permits unrestricted use, distribution, and reproduction in any medium, provided the original work is properly cited.

\begin{abstract}
This randomized trial proposed to determine if there were differences in calf muscle $\mathrm{StO}_{2}$ parameters in patients before and after 12 weeks of a traditional walking or walking-with-poles exercise program. Data were collected on 85 patients who were randomized to a traditional walking program $(n=40)$ or walking-with-poles program $(n=45)$ of exercise training. Patients walked for 3 times weekly for 12 weeks. Seventy-one patients completed both the baseline and the 12-week follow-up progressive treadmill tests ( $n=36$ traditional walking and $n=35$ walking-with-poles). Using the near-infrared spectroscopy measures, $\mathrm{StO}_{2}$ was measured prior to, during, and after exercise. At baseline, calf muscle oxygenation decreased from $56 \pm 17 \%$ prior to the treadmill test to $16 \pm 18 \%$ at peak exercise. The time elapsed prior to reaching nadir $\mathrm{StO}_{2}$ values increased more in the traditional walking group when compared to the walking-with-poles group. Likewise, absolute walking time increased more in the traditional walking group than in the walking-with-poles group. Tissue oxygenation decline during treadmill testing was less for patients assigned to a 12week traditional walking program when compared to those assigned to a 12-week walking-with-poles program. In conclusion, the 12-week traditional walking program was superior to walking-with-poles in improving tissue deoxygenation in patients with PAD.
\end{abstract}

\section{Introduction}

Walking ability is impaired in patients with peripheral arterial disease (PAD) due to decreased blood flow to the skeletal muscle tissue used during walking. It has been demonstrated that walking distance is associated with the degree of impairment in the affected leg as measured by the ankle-brachial index $(A B I)$ and the time to onset of claudication pain [1-6]. Likewise, it has been shown that walking exercise in patients with PAD prolongs the onset of claudication pain thus allowing the patient to walk longer but does not change the ABI [7-9].

Recently, several investigators have used near-infrared spectroscopy (NIRS) to dynamically study the effect of walking on oxygen desaturation of the gastrocnemius muscle of the impaired leg during exercise [2, 10-14]. Investigators noted a marked decline in skeletal muscle tissue oxygenation $\left(\mathrm{StO}_{2}\right)$ during walking exercise [2, 10-14]. Gardner et al. also identified that the rate of decline in calf muscle $\mathrm{StO}_{2}$ was significantly associated with initial claudication time and absolute claudication time in patients with PAD [2]. Three of the above studies examined the changes in tissue oxygenation, using NIRS, in patients with PAD before and after an exercise training program [12-14]. Figoni et al. [12] used a pretest-post-test design and reported greater exercise duration and lower mean exercise $\mathrm{StO}_{2}$ in 15 patients after a 3-month treadmill and calf exercise training program. Manfredini et al. [13] studied 55 patients with PAD who were given the option to participate in a structured or unstructured home exercise program for $34 \pm 2$ weeks. 
At the end of the study, those patients in the structured exercise program had an increased oxyhemoglobin area under the curve when compared to baseline; similar changes were not identified in the unstructured exercise group. Lastly, Tew et al. [11] studied calf-tissue oxygenation and treadmill walking response time after randomization to 12 weeks of arm-crank ergometry or a control group in 57 patients with intermittent claudication. Investigators identified that walking distance and time to nadir $\mathrm{StO}_{2}$ increased in those assigned to arm-cranking and there were no changes in the control group. No studies however compared leg muscle responses after a traditional walking program compared to a walking-with-poles program of rehabilitation in patients with claudication.

The purpose of this study was to determine if there were differences in calf muscle $\mathrm{StO}_{2}$ parameters before and after 12 weeks of a traditional walking program versus a walkingwith-poles exercise program. We reasoned that by using the poles to offload the legs, patients would have less claudication pain and be able to walk longer distance thus achieving a greater training effect. We hypothesized that the calf muscle tissue deoxygenation would be less in both groups after 12 weeks of exercise training than at baseline and that the decrease would be greater in those patients assigned to the walking-with-poles group. These data represent a subanalysis of data from a larger clinical trial comparing traditional walking to walking with poles [14].

\section{Methods}

2.1. Subjects. Patients with PAD were screened and recruited to participate in a randomized clinical trial designed to compare the effects of a traditional walking training program versus walking-with-poles exercise training program. A total of 2296 patients were screened for eligibility, 146 enrolled in the study [14]. Patients were recruited using radio and newspaper advertising, posted fliers, and letters of invitation sent to patients at the university and VA hospitals. For the purposes of this analysis, we included only those participants with an $\mathrm{ABI}$ of $\leq 0.90$ ( $n=91$ patients). Of those 91 patients, 3 did not have $\mathrm{StO}_{2}$ analyses on their baseline progressive treadmill test due to equipment malfunction and 3 had questionable data resulting in a final sample of 85 patients. Of those 85 patients, 45 were assigned to the walking-withpoles group and 40 patients were assigned to the traditional walking group.

2.2. Research Design. The major study was a randomized clinical trial to examine the effects of a traditional walking program compared to walking with poles on walking endurance and perceived physical function in patients with PAD. This subanalysis provides an in-depth examination of $\mathrm{StO}_{2}$ responses to exercise training. Our primary outcome focused on changes the patients realized walking on the constant work rate treadmill test whereas this analysis used data from the progressive treadmill test. At two minutes on the constant work rate test, the speed and incline increased to $85 \%$ of the patient's workload on the progressive treadmill test resulting in a sudden decline in $\mathrm{StO}_{2}$. Due to the gentle nature of the progressive treadmill protocol used, we were able to detect more subtle changes in $\mathrm{StO}_{2}$ and onset of claudication pain.

The intervention consisted of traditional walking or walking with poles three times weekly. Patients were randomized using computer-generated permuted blocks and group assignment was managed by the study statistician. The training programs for both groups were identical except that one group exercised with poles and the other group did not. Patients assigned to the walking-with-poles group received training on the use of the poles prior to beginning the exercise program. Additionally, patients were coached on proper poling mechanics during the training period if needed. The training program incorporated interval training whereby exercise consisted mostly of low-tomoderate intensity training at the start of the program and progressed to moderate-to-high intensity. Exercise intensity was determined by heart rate associated with a percentage of oxygen uptake obtained during the metabolic exercise treadmill tests. Exercise duration and intensity was adjusted every three weeks. During the first weeks, patients walked for 30 minutes (20\% light intensity, $60 \%$ moderate intensity, and $20 \%$ hard intensity). By weeks $10-12$, patients walked for 55 minutes (10\% light intensity, $45 \%$ moderate intensity, and $45 \%$ hard intensity). Light intensity was defined as $25-$ $44 \%$ peak $\mathrm{VO}_{2}$, moderate intensity was $45-59 \%$ peak $\mathrm{VO}_{2}$, and hard intensity was $60-84 \% \mathrm{VO}_{2}$ peak. The program was designed so that patients walked on the treadmill two days/week and outdoors or in the hospital corridors one day/week. The exercise interval was adjusted based on the patient's pain tolerance. For example, some patients could only walk for one to two minutes at the higher intensities. In order for them to achieve $20 \%$ total time at the harder intensities, multiple short intervals were completed. Exercise intervals were gradually lengthened and rest times between intervals were gradually decreased.

2.3. Measurements. Demographic information, height, weight, comorbid conditions, and medication history were obtained from the patient and reviewed in the patient medical record. A physical exam was completed prior to participation in the study.

2.3.1. Ankle-Brachial Index. The ABI was measured in the more severely affected leg at baseline and 12 weeks after training. The dorsalis pedis or posterior tibial arteries were used for measurement. The location of the signal was recorded and used consistently throughout the study for that individual subject. After the patient rested comfortably in a supine position for 15 minutes, Doppler ultrasound was used to measure the systolic pressure in the right and left arms and in the ankle of the most severely affected leg. The arm with the highest systolic pressure was used to calculate the ABI [15].

2.3.2. Symptom-Limited Treadmill Test Protocol. Patients were tested using a gentle treadmill protocol that was developed for patients with PAD $[6,16]$. Increases in percent grade occurred every 30 seconds, and, after the first 6 minutes, 
TABle 1: Demographic characteristics of the sample.

\begin{tabular}{|c|c|c|c|c|}
\hline Characteristic & $\begin{array}{c}\text { Combined } \\
n=85\end{array}$ & $\begin{array}{l}\text { Walking with poles } \\
\qquad n=45\end{array}$ & $\begin{array}{l}\text { Traditional walking } \\
\qquad n=40\end{array}$ & $P$ value \\
\hline Age, years & $69.4 \pm 9.1$ & $71.7 \pm 9.2$ & $66.8 \pm 8.5$ & .012 \\
\hline $\mathrm{ABI}$ & $0.63 \pm 0.18$ & $0.62 \pm 0.20$ & $0.63 \pm 0.17$ & .75 \\
\hline Weight, kg & $89.6 \pm 16.4$ & $89.1 \pm 14.4$ & $90.4 \pm 18.7$ & .71 \\
\hline BMI, $\mathrm{kg} / \mathrm{m}^{2}$ & $29.0 \pm 4.9$ & $29.2 \pm 4.4$ & $28.9 \pm 5.5$ & .77 \\
\hline ICT, min & $4.45 \pm 3.67$ & $4.18 \pm 3.34$ & $4.70 \pm 3.98$ & .32 \\
\hline AWT, min & $8.47 \pm 4.05$ & $8.58 \pm 3.92$ & $8.35 \pm 4.22$ & .77 \\
\hline Peak oxygen uptake, $\mathrm{ml} \cdot \mathrm{kg}^{-1} \cdot \mathrm{min}^{-1}$ & $14.3 \pm 2.9$ & $14.0 \pm 2.7$ & $14.6 \pm 3.1$ & .39 \\
\hline Sex, \% male & $93 \%$ & $91 \%$ & $95 \%$ & .66 \\
\hline Race, \% caucasian & $78 \%$ & $80 \%$ & $77 \%$ & .78 \\
\hline Current smoking, \% yes & $35 \%$ & $36 \%$ & $33 \%$ & .66 \\
\hline Diabetes, $\%$ yes & $49 \%$ & $47 \%$ & $52 \%$ & .93 \\
\hline Hypertensive, \% yes & $85 \%$ & $78 \%$ & $92 \%$ & .54 \\
\hline Dyslipidemia, \% yes & $74 \%$ & $76 \%$ & $73 \%$ & .79 \\
\hline
\end{tabular}

AWT: absolute walking time; ICT: initial claudication time.

speed increased every 3 minutes. Validity and reliability of the treadmill protocol has been previously reported [17]. Electrocardiogram and $\mathrm{StO}_{2}$ of the gastrocnemius muscle were monitored continuously throughout the test. Initial claudication time was defined as the time during the treadmill test when the subject experienced pain in the affected leg. The absolute walking time was defined as the time walked by the patient on the progressive treadmill test.

2.3.3. Metabolic Values. Peak oxygen uptake was measured using the MedGraphics CPX/D System (Medical Graphics Corp, St. Paul, MN, USA). The metabolic cart was calibrated using a $3 \mathrm{~L}$ syringe prior to each test and the analyzers were calibrated using references gasses and room air. Patients breathed through a mouthpiece and their nose was clipped with a standard nose clip. Oxygen uptake was averaged over $30 \mathrm{~s}$ and the highest value at peak exercise was recorded.

2.3.4. Near-Infrared Spectroscopy Measures (NIRS). Skeletal muscle tissue oxygenation was measured prior to, during, and after exercise using the NIRS spectrometer (InSpectra 325, Hutchinson Technology, Inc., Hutchinson, MN, USA). A $25 \mathrm{~mm}$ probe attached to an optical cable was used to noninvasively measure the percentage of hemoglobin oxygen saturation of the tissue beneath the near-infrared light. The InSpectra machine was calibrated prior to each test according to known high and low reference standards [18]. The NIRS probe was attached to the medial section of the gastrocnemius muscle of the patient's most severely affected leg. Calf circumference measurements were taken to assure consistent placement of the probe. The probe was placed into a specially designed adhesive housing unit to reduce ambient light. The probe was then secured to the leg to avoid excessive motion and possible tripping. The $\mathrm{StO}_{2}$ measures were recorded every 3.5 seconds using the InSpectra software. The $\mathrm{StO}_{2}$ values used in this analysis were the $\mathrm{StO}_{2}$ value recorded after 2 minutes of standing prior to exercise $\left(\mathrm{StO}_{2}\right.$ rest), time walked when the patient reached the nadir $\mathrm{StO}_{2}$ value (time to nadir $\mathrm{StO}_{2}$ value), $\mathrm{StO}_{2}$ value at peak exercise $\left(\mathrm{StO}_{2}\right.$ peak), and absolute and relative drop in $\mathrm{StO}_{2}$ (absolute drop in $\mathrm{StO}_{2}=$ rest $\mathrm{StO}_{2}-$ minimal $\mathrm{StO}_{2}$ value, relative drop in $\mathrm{StO}_{2}=\left[\right.$ rest $\mathrm{StO}_{2}-$ nadir $\mathrm{StO}_{2}$ value $] /$ rest $\left.\mathrm{StO}_{2}\right)$.

2.3.5. Ratings of Perceived Pain. Ratings of perceived leg pain were obtained every minute using the Borg ratio scale [19]. Patients were instructed to notify the staff at the onset of leg pain. The initial claudication time is the time that patients either notified the staff of the onset of pain or the time they first reported pain when asked every minute.

2.4. Statistical Analysis. Measures of central tendency were completed to describe the sample. The two groups were compared at baseline using the $t$-test for independent samples and Mann Whitney $U$ test. Data that were not normally distributed were log transformed. Regression analyses were used to compute the initial decline in slopes for $\mathrm{StO}_{2}$ values $\left(\mathrm{StO}_{2}\right.$ value regressed over time). Pearson $\mathrm{r}$ correlations were completed to examine relationships between the variables. Paired $t$-tests were used to describe changes in measures from baseline to post-12 weeks of training. Independent $t$-tests were used to examine differences between groups. Intentto-treat analyses using the baseline value carried forward were completed for the comparisons between groups. The criterion for significance was $P<.05$.

\section{Results}

The sample consisted of primarily older (age $=69.4 \pm 9.2$ years) men. The ABI was $.63 \pm .19$. Over one-third of the patients were current smokers, half were diabetic, and the majority of patients received treatment for hypertension and dyslipidemia (Table 1). Patients were generally unfit with a peak oxygen uptake of $14.3 \pm 2.9 \mathrm{~mL} \cdot \mathrm{kg}^{-1} \cdot \mathrm{min}^{-1}$. The walking-with-poles group was older than the traditional walking group. No other differences in baseline demographic characteristics were identified. 
TAвLE 2: Relationships between selected variables and initial claudication and absolute walking times $(n=85)$.

\begin{tabular}{|c|c|c|c|c|}
\hline Variables & ICT Pearson $r$ & $P$ value & AWT Pearson $r$ & $P$ value \\
\hline $\mathrm{StO}_{2}$ at rest & .07 & .51 & .02 & .87 \\
\hline Peak exercise $\mathrm{StO}_{2}$ & .14 & .22 & .23 & .04 \\
\hline Absolute decline in $\mathrm{StO}_{2}$ during exercise & -.19 & .08 & -.12 & .30 \\
\hline Relative decline in $\mathrm{StO}_{2}$ during exercise & -.27 & .01 & -.17 & .14 \\
\hline Time walked to nadir $\mathrm{StO}_{2}$ value & .42 & .000 & .58 & .000 \\
\hline Recovery time to baseline $\mathrm{StO}_{2}$ & -.31 & .02 & .26 & .04 \\
\hline Recovery $\mathrm{StO}_{2}$ at $1 \mathrm{~min}$ & .37 & .001 & .31 & .01 \\
\hline Recovery $\mathrm{StO}_{2}$ at $3 \mathrm{~min}$ & .36 & .001 & .37 & .001 \\
\hline Recovery $\mathrm{StO}_{2}$ at $5 \mathrm{~min}$ & .28 & .01 & .36 & .001 \\
\hline Exercise slope & .28 & .01 & .18 & .11 \\
\hline Recovery slope & .18 & .11 & .21 & .07 \\
\hline
\end{tabular}

AWT: absolute walking time; ICT: initial claudication time.

At baseline, calf muscle oxygenation decreased from $56 \pm 17 \%$ prior to the treadmill test to $16 \pm 18 \%$ at peak exercise (71\% relative decline from rest to peak exercise). Patients walked $4.45 \pm 3.67$ minutes before experiencing claudication pain and $4.39 \pm 3.99$ minutes when the $\mathrm{StO}_{2}$ reached its nadir value. There was no difference between the time elapsed when the $\mathrm{StO}_{2}$ reached its nadir value and pain onset $(P=.87)$. Although all patients reported claudication pain, exercise was not limited by claudication pain for all patients. Therefore, we examined the onset of claudication pain and time to nadir $\mathrm{StO}_{2}$ for those whose exercise was limited by claudication pain only $(n=72)$. Patients walked $3.35 \pm 2.57$ minutes before experiencing claudication pain and $3.67 \pm 3.49$ minutes when the $\mathrm{StO}_{2}$ reached its nadir value. There was no difference between the time elapsed when the $\mathrm{StO}_{2}$ reached its nadir value and pain onset $(P=.40)$. After exercise, the time for the $\mathrm{StO}_{2}$ to return to half of its resting value was $1.3 \pm 1.3 \mathrm{~min}$ and $2.2 \pm 2.1$ minutes to return to resting values. The initial claudication time and absolute walking time were not associated with the $\mathrm{StO}_{2}$ values at rest (Table 2) but were moderately related to the time elapsed prior to reaching the nadir $\mathrm{StO}_{2}$ value $(r=.42, P<.001 ; r=.58, P=.001$, resp. $)$. There was also a small to moderate relationship between onset of claudication pain and the exercise slope decline in $\mathrm{StO}_{2}(r=$ $.28, P=.011)$. Relationships were similar between initial claudication time and absolute walking time and the time elapsed prior to reaching the nadir $\mathrm{StO}_{2}$ values when the analysis was restricted to those whose exercise was limited by claudication $(r=48, P \leq .001$ and $r=.47, P<.001)$.

Data comparing groups after exercise training were analyzed using intent-to-treat analysis for all patients. The change in time elapsed prior to reaching nadir $\mathrm{StO}_{2}$ values between baseline and 12 weeks was related to the change in absolute walking time between baseline and 12 weeks $(r=$ $.65, P<.001)$ and initial claudication time at 12 weeks $(r=$ .33, $P=.002$ ). Analysis of covariance was completed to compare the groups with age as the covariate since there was a difference in age at baseline (Table 3 ). The change in the time elapsed prior to reaching nadir $\mathrm{StO}_{2}$ values $(P=.002)$ and absolute walking time $(P=.002)$ were greater in the

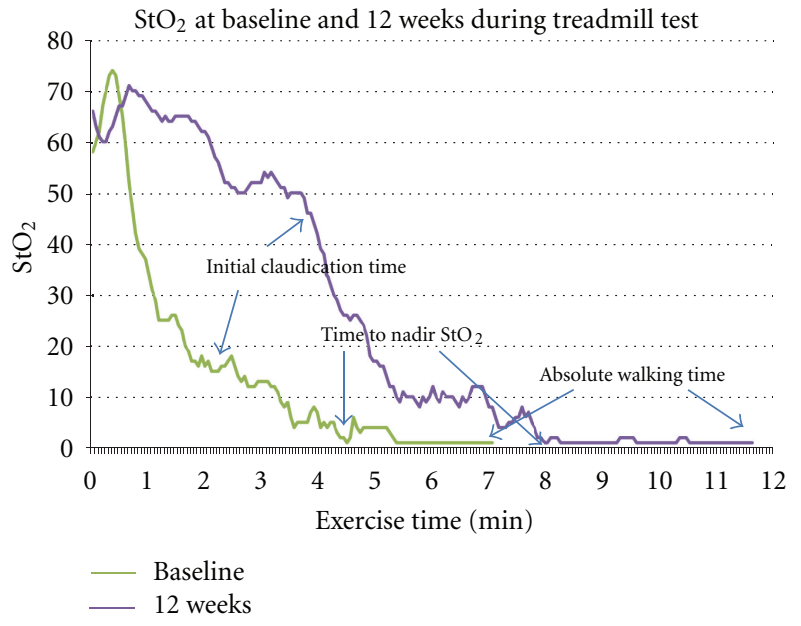

Figure 1: $\mathrm{StO}_{2}$ data from sample patient at baseline and 12 weeks.

traditional walking group when compared to the walkingwith-poles group. There was, however, no difference in the change in initial claudication time between the two groups $(P=.38)$.

When the analyses were restricted to those who completed the exercise training only $(n=71)$, the change in time elapsed prior to reaching nadir $\mathrm{StO}_{2}$ values between baseline and 12 weeks was related to the change in absolute walking time between baseline and 12 weeks $(r=.57, P<$ $.001)$ and initial claudication time at 12 weeks $(r=.32$, $P=.004)$. Data from a sample patient are depicted in Figure 1. Recovery time was unchanged when baseline and 12 -week values were compared (baseline $=2.45 \pm 2.17 \mathrm{~min}$, $12 \mathrm{wk}=2.19 \pm 1.94 \mathrm{~min}, P=.81)$. After 12 weeks of exercise training, within-group changes in initial claudication time, absolute claudication time and time elapsed prior to reaching nadir $\mathrm{StO}_{2}$ values increased (Table 4.) The time elapsed prior to reaching nadir $\mathrm{StO}_{2}$ values increased more in the traditional walking group when compared to the walkingwith-poles group (walking group $=4.01 \pm 5.13$ minutes, walking-with-poles group $=.89 \pm 3.56$ minutes, $P=.009$ ). 
TABLE 3: Exercise times at baseline and 12 weeks, intent-to-treat analysis $(n=85)$.

\begin{tabular}{|c|c|c|c|c|c|}
\hline & \multicolumn{2}{|c|}{ Traditional walking group } & \multicolumn{2}{|c|}{ Walking-with-poles group } & \multirow[b]{2}{*}{$\begin{array}{c}\text { Between group } \\
P \text { value }\end{array}$} \\
\hline & $\begin{array}{l}\text { Walking time minutes } \\
\begin{array}{c}(\text { mean } \pm \mathrm{SD}) \\
n=40\end{array}\end{array}$ & $\begin{array}{l}\text { Within group } \\
\quad P \text { value }\end{array}$ & $\begin{array}{l}\text { Walking time minutes } \\
\qquad \begin{array}{c}(\text { mean } \pm \mathrm{SD}) \\
n=45\end{array}\end{array}$ & $\begin{array}{l}\text { Within group } \\
\quad P \text { value }\end{array}$ & \\
\hline \multicolumn{6}{|c|}{ Initial claudication time } \\
\hline (i) Baseline & $3.95 \pm 2.97$ & .034 & $3.33 \pm 2.75$ & .15 & \\
\hline (ii) $12 \mathrm{wk}$ & $5.23 \pm 4.29$ & & $3.98 \pm 3.50$ & & \\
\hline (iii) Change & $1.28 \pm 3.68$ & & $0.64 \pm 2.94$ & & .38 \\
\hline \multicolumn{6}{|c|}{ Absolute walking time } \\
\hline (i) Baseline & $8.01 \pm 4.01$ & .000 & $7.76 \pm 4.01$ & .92 & \\
\hline (ii) $12 \mathrm{wk}$ & $11.30 \pm 5.14$ & & $7.83 \pm 5.57$ & & \\
\hline (iii) Change & $3.73 \pm 3.15$ & & $1.74 \pm 2.42$ & & .002 \\
\hline \multicolumn{6}{|c|}{ Time to nadir value } \\
\hline (i) Baseline & $4.25 \pm 4.16$ & .000 & $3.69 \pm 3.07$ & .22 & \\
\hline (ii) $12 \mathrm{wk}$ & $7.94 \pm 5.89$ & & $4.30 \pm 3.87$ & & \\
\hline (iii) Change & $3.59 \pm 5.19$ & & $0.60 \pm 3.23$ & & .002 \\
\hline
\end{tabular}

Time to nadir value: time elapsed to reach nadir $\mathrm{StO}_{2}$ value. Above analyses were adjusted for age.

TABLE 4: Exercise times at baseline and 12 weeks for those who completed training $(n=71)$.

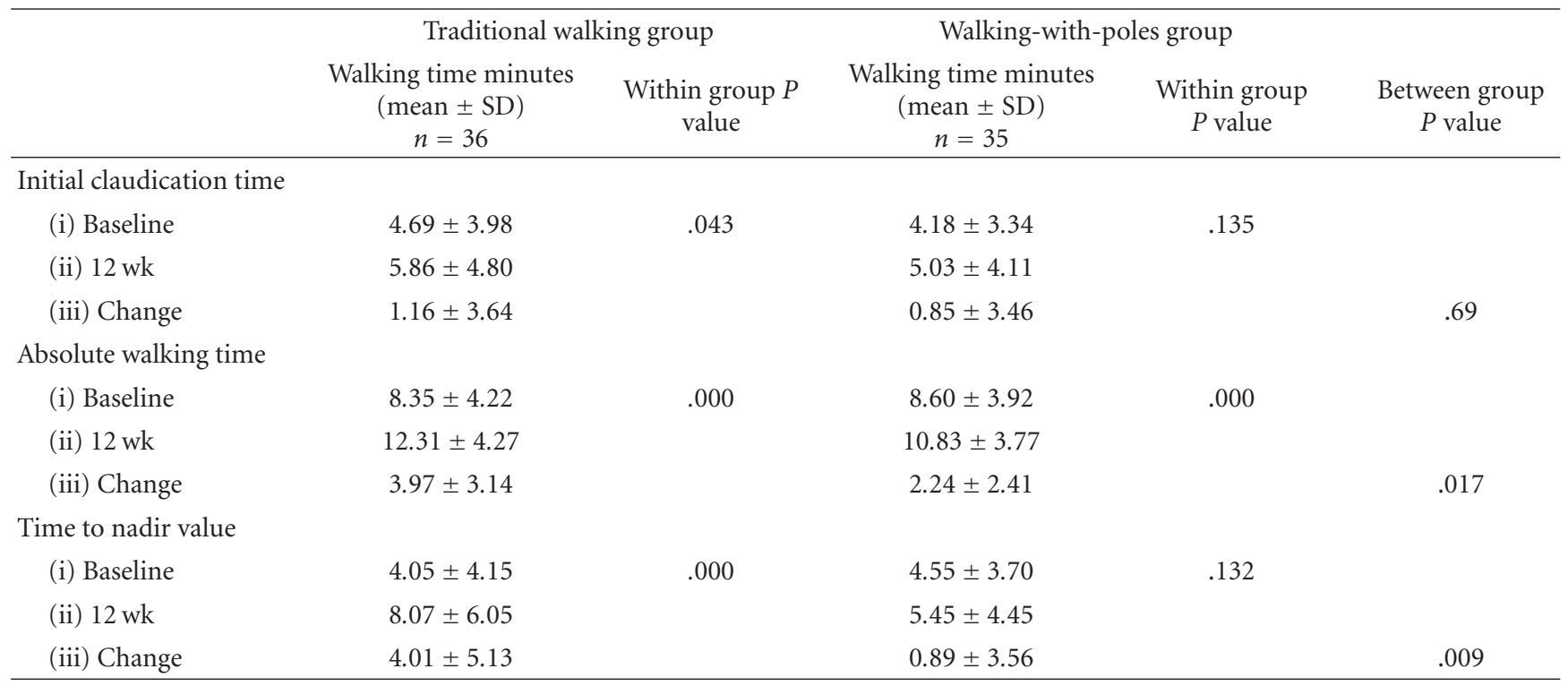

Time to nadir value: time elapsed to reach nadir $\mathrm{StO}_{2}$ value. Above analyses were adjusted for age.

Similarly, absolute walking time increased more in the traditional walking group than in the walking-with-poles group (walking group $=3.97 \pm 3.14$ minutes, walking-withpoles group $=2.24 \pm 2.41$ minutes, $P=.017$ ). There was no difference between the two groups in initial claudication time (walking group $=1.16 \pm 3.64$ minutes, walking-withpoles group $=.85 \pm 3.46$ minutes, $P=.69$ ). Since there was a significant difference in age between the two groups, age was entered as a covariate yet it did not contribute significantly to the model. No differences in recovery time were noted between the traditional walking and walkingwith-poles groups at the 12-week follow-up time period (traditional walking recovery time $=1.26 \pm 1.43 \mathrm{~min}$, walking with poles $=1.41 \pm 1.76 \mathrm{~min}, P=.70)$. No significant differences were identified in peak oxygen consumption or $\mathrm{ABI}$ within or between the two groups from baseline to 12 weeks using intent-to-treat analysis or restricting the analysis to those who completed the training only.

\section{Discussion}

There are three major findings of this study: (1) the time elapsed before reaching nadir $\mathrm{StO}_{2}$ values is associated with initial claudication time and absolute walking time, (2) there 
was no difference in the time associated with the nadir of $\mathrm{StO}_{2}$ and the onset of claudication pain, and (3) the time to nadir $\mathrm{StO}_{2}$ of the gastrocnemius muscle was greater after 12 weeks of exercise training in the traditional walking group when compared to the walking with poles group.

As Bauer notes, during higher work rates and apparent blood flow limitation, muscle oxygen desaturation becomes more rapid in PAD subjects [19]. Thus, one would expect that oxygen desaturation would be associated with onset of claudication and walking duration. Our findings reflect these assumptions and were similar to those reported by Gardner et al. [2], that is, shorter time to reach nadir $\mathrm{StO}_{2}$ values were associated with shorter initial claudication time and total walking duration. Second, in Gardner's study, relationships between initial claudication time $(r=.339)$, absolute walking time $(r=.68)$ and measured time to minimal exercise $\mathrm{StO}_{2}$ were similar to our relationships between initial claudication time $(r=.42)$, absolute walking time $(r=.58)$ and measured distance to nadir $\mathrm{StO}_{2}$. These findings suggest that our sample patients were similar to patients in other studies $[2,19]$.

Exercise-associated decline in $\mathrm{StO}_{2}$ of the gastrocnemius muscle was less after 12 weeks of exercise training when compared to baseline. Wang and colleagues recently demonstrated in 17 subjects with PAD who completed a 24-week walking program that an increase in the number of capillaries in contact with type IIx and IIa calf muscle fibers was related to an improved pain-free walking time $(r=.69$ and $r=.62$, $P<.05)[8]$. Investigators hypothesized that the ratio of capillaries to muscle fibers affects oxygen supply thus delaying the mismatch between supply and demand [8]. Figoni et al. [12] and Manfredini et al. [13] both report greater exercise $\mathrm{StO}_{2}$ area under the curve postexercise training when compared to preexercise training. In our study, an increase in the time to nadir $\mathrm{StO}_{2}$ values was associated with delayed initial claudication time and improved absolute walking time. Our findings provide further evidence that walking exercise improves tissue muscle oxygenation in patients with PAD. Likewise, our findings support the possibility that the onset of claudication and walking duration are linked by the decline in tissue oxygenation. The onset of claudication and the time elapsed prior to reaching the nadir $\mathrm{StO}_{2}$ values were nearly identical. Thus, the patient's perception of pain is a good indicator of $\mathrm{StO}_{2}$ in the calf muscle.

Exercise-induced decline in gastrocnemius muscle $\mathrm{StO}_{2}$ was less in patients assigned to the traditional walking program than in patients assigned to the walking-with-poles exercise training program. We had originally hypothesized that a walking training program with poles for patients would be advantageous because the poles would "offload" the legs during periods of claudication allowing additional walking exercise. Indeed, Oakley et al. [20] investigated the immediate effects of walking with Nordic poles on 20 patients with PAD and found that claudication distance increased from $77 \mathrm{~m}$ without the poles to $130 \mathrm{~m}$ with the poles $(P<.0001)$ and the level of claudication pain also decreased $(P=.0002)$. Our group has also previously reported that patients had decreased calf pain when walking with poles. Oakley et al. [20] compared muscle histology of 36 patients randomly assigned to 12 weeks of treadmill training, strength training or control. After the 12 weeks, only the treadmill group had histological changes in the muscle. The increase in muscle ischemia with treadmill training was associated with a marked increase in muscle damage. Hiatt suggests that skeletal muscle dysfunction and associated muscle metabolic state can be ameliorated with treadmill exercise training. Tew et al. [11] provides evidence that even when the exercising muscle is not the calf muscle, tissue oxygenation can improve. Our evidence is equivocal. Clearly, the patients assigned to the traditional walking group experienced a prolonged exercise time before experiencing claudication pain, prolonged their absolute walking time and increased the amount of time exercising until reaching nadir values. In the walking-with-poles group, there were no changes over time when an intent-to-treat approach was used. However, when the analysis was restricted to those who completed the training program only, there was a difference in absolute walking time and a trend toward increasing time to initial claudication pain onset as well as nadir $\mathrm{StO}_{2}$. The explanation for these findings may be that leg metabolic changes in the calf muscle occurred to a greater extent in those assigned to the walking-with-poles group or we may not have had enough statistical power to detect changes that were present.

Another explanation for this finding may be that the intensity of our training program was based on oxygen uptake associated with heart rate response to exercise. With the addition of the upper body to the exercise regime for patients assigned to the walking-with-poles group, exercise intensity was augmented using the upper body. Thus, the work accomplished by the leg muscles may have been less even though overall exercise intensity was consistent between the two groups of patients. Both groups trained on the treadmill and outdoors. Patients assigned to the walking with poles group were unable to use the handrails on the treadmill for balance; added anxiety may have increased the cardiovascular response thus keeping the overall work accomplished lower in some patients.

Our study had several limitations that may limit the generalizability of the findings. First, although age was not a contributing factor to the differences between the groups, the walking-with-poles group was older than the traditional walking group. We cannot exclude that the differences in exercise tolerance and willingness to push limits may differ between someone who is 67 years old (mean age for walking group) and someone who is 72 years old (mean age for walking-with-poles group). This five-year age difference may have influenced our outcomes in ways that we did not measure. Next, the sample was primarily men and thus cannot be generalized to women. Additionally, in order to include only those with compressible vessels, we excluded 17 patients who were initially randomized to our study. Although this limited the variability, it also reduced power in our statistical testing. Lastly, although all of our patients experienced claudication pain, not all patients stopped exercise due to claudication pain. Including patients that did not stop exercise due to claudication pain may have affected the study results. 
Additional studies examining different walking intensities in patients with peripheral arterial disease should be tested. Further, it may be that use of walking poles early in the rehabilitative effort may augment traditional walking training later by assisting patients to train longer early on. Further research in this area is warranted.

\section{Conclusion}

In summary, we conclude that there is a significant decline in $\mathrm{StO}_{2}$ in the gastrocnemius muscle during walking exercise in patients with peripheral arterial disease reflecting muscle tissue ischemia. Contrary to our hypothesis, after 12 weeks of exercise training, absolute walking time and time to reach nadir muscle tissue oxygenation were prolonged in the patients assigned to the traditional walking program more than those assigned to a walking-with-poles exercise training program reflecting greater tissue perfusion and enhancement of arteriovenous oxygen extraction. The precise mechanism for these differences remains unknown but these findings may provide some insight to tissue adaptations to exercise stimulus and may therefore be useful to PAD rehabilitation programs.

\section{Abbreviations}

ABI: Ankle-brachial index

AWT: Absolute walking time

ICT: Initial claudication time

NIRS: Near-infrared spectroscopy

PAD: Peripheral arterial disease

$\mathrm{StO}_{2}$ : Skeletal muscle tissue oxygenation.

\section{Authors' Contribution}

E. G. Collins and D. Reda are responsible for the concept and design. E. G. Collins. C. McBurney, J. Butlur, C. Jelinek, and S. O'Connell are responsible for the acquisition of data. E. G. Collins supervised the study. The drafting of paper was carried out by E. G. Collins, C. McBurney, J. Butlur, C. Jelinek, S. O'Connell,C. Fritschi, and D. Reda. The critical revision of the paper for important intellectual content (principal investigation) the principal investigator E. G. Collins obtained funding. E. G. Collins and D. Reda are responsible for administrative, technical, or material support.

\section{Conflict of Interests}

The authors have declared that no competing interests exist.

\section{Disclosure}

This study was approved by the Institutional Review Boards at the University of Illinois at Chicago and the Edward Hines Jr., VA Hospital. Written informed consent was obtained prior to enrolling patients in the study.

\section{Acknowledgments}

This material was based on work supported by a Grant from the National Institute of Nursing Research (RO1 NR00887701) and a Research Career Scientist Award (E. G. Collins), Rehabilitation Research \& Development, Department of Veterans Affairs. The authors acknowledge the editorial assistance of Mary Reidy.

\section{References}

[1] B. Sanderson, C. Askew, I. Stewart, P. Walker, H. Gibbs, and S. Green, "Short-term effects of cycle and treadmill training on exercise tolerance in peripheral arterial disease," Journal of Vascular Surgery, vol. 44, no. 1, pp. 119-127, 2006.

[2] A. W. Gardner, D. E. Parker, N. Webb, P. S. Montgomery, K. J. Scott, and S. M. Blevins, "Calf muscle hemoglobin oxygen saturation characteristics and exercise performance in patients with intermittent claudication," Journal of Vascular Surgery, vol. 48, no. 3, pp. 644-649, 2008.

[3] M. M. McDermott, K. Liu, J. M. Guralnik, G. J. Martin, M. H. Criqui, and P. Greenland, "Measurement of walking endurance and walking velocity with questionnaire: validation of the walking impairment questionnaire in men and women with peripheral arterial disease," Journal of Vascular Surgery, vol. 28, no. 6, pp. 1072-1081, 1998.

[4] A. W. Gardner, R. M. Ritti-Dias, J. A. Stoner, P. S. Montgomery, K. J. Scott, and S. M. Blevins, "Walking economy before and after the onset of claudication pain in patients with peripheral arterial disease," Journal of Vascular Surgery, vol. 51, no. 3, pp. 628-633, 2010.

[5] M. M. McDermott, J. M. Guralnik, L. Ferrucci et al., "Asymptomatic peripheral arterial disease is associated with more adverse lower extremity characteristics than intermittent claudication," Circulation, vol. 117, no. 19, pp. 2484-2491, 2008.

[6] E. G. Collins, W. E. Langbein, C. Orebaugh et al., "PoleStriding exercise and vitamin E for management of peripheral vascular disease," Medicine and Science in Sports and Exercise, vol. 35, no. 3, pp. 384-393, 2003.

[7] A. W. Gardner, L. I. Katzel, J. D. Sorkin et al., "Exercise rehabilitation improves functional outcomes and peripheral circulation in patients with intermittent claudication: a randomized controlled trial," Journal of the American Geriatrics Society, vol. 49, no. 6, pp. 755-762, 2001.

[8] J. Wang, S. Zhou, R. Bronks, J. Graham, and S. Myers, "Effects of supervised treadmill walking training on calf muscle capillarization in patients with intermittent claudication," Angiology, vol. 60, no. 1, pp. 36-41, 2009.

[9] T. P. Murphy, D. E. Cutlip, J. G. Regensteiner, E. R. Mohler, D. J. Cohen, M. R. Reynolds et al., "Supervised exercise versus primary stenting for claudication resulting from aortoiliac peripheral artery disease: six-month outcomes from the claudication: exercise versus endoluminal revascularization (CLEVER) study," Circulation, vol. 125, no. 1, pp. 130-139, 2012.

[10] F. Manfredini, A. M. Malagoni, M. Felisatti et al., "A dynamic objective evaluation of peripheral arterial disease by nearinfrared spectroscopy," European Journal of Vascular and Endovascular Surgery, vol. 38, no. 4, pp. 441-448, 2009.

[11] G. Tew, S. Nawaz, I. Zwierska, and J. M. Saxton, "Limb-specific and cross-transfer effects of arm-crank exercise training in 
patients with symptomatic peripheral arterial disease," Clinical Science, vol. 117, no. 12, pp. 405-413, 2009.

[12] S. F. Figoni, C. F. Kunkel, A. M. E. Scremin et al., "Effects of exercise training on calf tissue oxygenation in men with intermittent claudication," Physical Medicine and Rehabilitation, vol. 1, no. 10, pp. 932-940, 2009.

[13] F. Manfredini, A. M. Malagoni, S. Mandini et al., "Nearinfrared spectroscopy assessment following exercise training in patients with intermittent claudication and in untrained healthy participants," Vascular and Endovascular Surgery, vol. 46, no. 4, pp. 315-324, 2012.

[14] E. G. Collins, S. O’Connell, C. McBurney et al., "Comparison of walking with poles and traditional walking for peripheral arterial disease rehabilitation," Journal of Cardiopulmonary Rehabilitation and Prevention, vol. 32, no. 4, pp. 210-218, 2012.

[15] S. M. Grenon, J. Gagnon, and Y. Hsiang, "Ankle-brachial index for assessment of peripheral arterial disease," The New England Journal of Medicine, vol. 361, no. 19, pp. e40.1-e40.3, 2009.

[16] W. E. Langbein, E. G. Collins, C. Orebaugh et al., "Increasing exercise tolerance of persons limited by claudication pain using polestriding," Journal of Vascular Surgery, vol. 35, no. 5, pp. 887-893, 2002.

[17] E. G. Collins, W. E. Langbein, C. Orebaugh et al., "Cardiovascular training effect associated with polestriding exercise in patients with peripheral arterial disease," The Journal of Cardiovascular Nursing, vol. 20, no. 3, pp. 177-185, 2005.

[18] T. A. Bauer, E. P. Brass, T. J. Barstow, and W. R. Hiatt, "Skeletal muscle $\mathrm{StO}_{2}$ kinetics are slowed during low work rate calf exercise in peripheral arterial disease," European Journal of Applied Physiology, vol. 100, no. 2, pp. 143-151, 2007.

[19] G. A. V. Borg, "Psychophysical bases of perceived exertion," Medicine and Science in Sports and Exercise, vol. 14, no. 5, pp. 377-381, 1982.

[20] C. Oakley, I. Zwierska, G. Tew, J. D. Beard, and J. M. Saxton, "Nordic poles immediately improve walking distance in patients with intermittent claudication," European Journal of Vascular and Endovascular Surgery, vol. 36, no. 6, pp. 689694, 2008. 


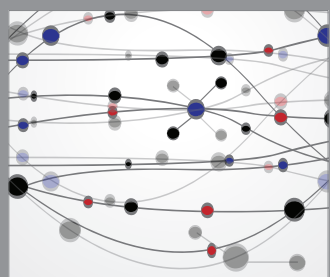

The Scientific World Journal
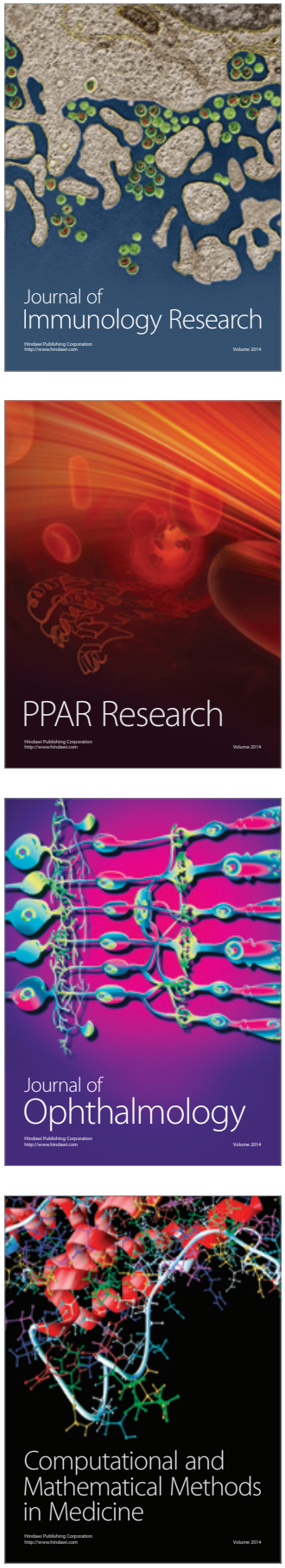

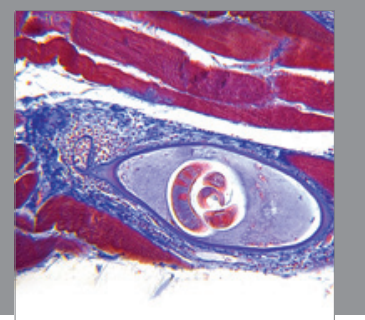

Gastroenterology

Research and Practice
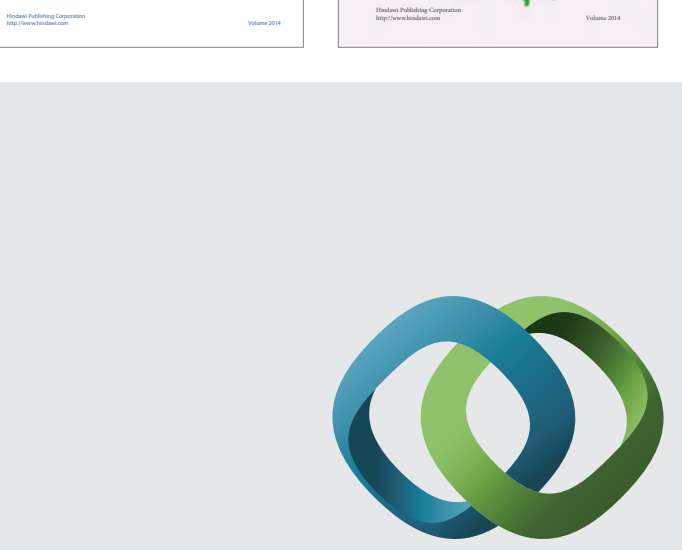

\section{Hindawi}

Submit your manuscripts at

http://www.hindawi.com
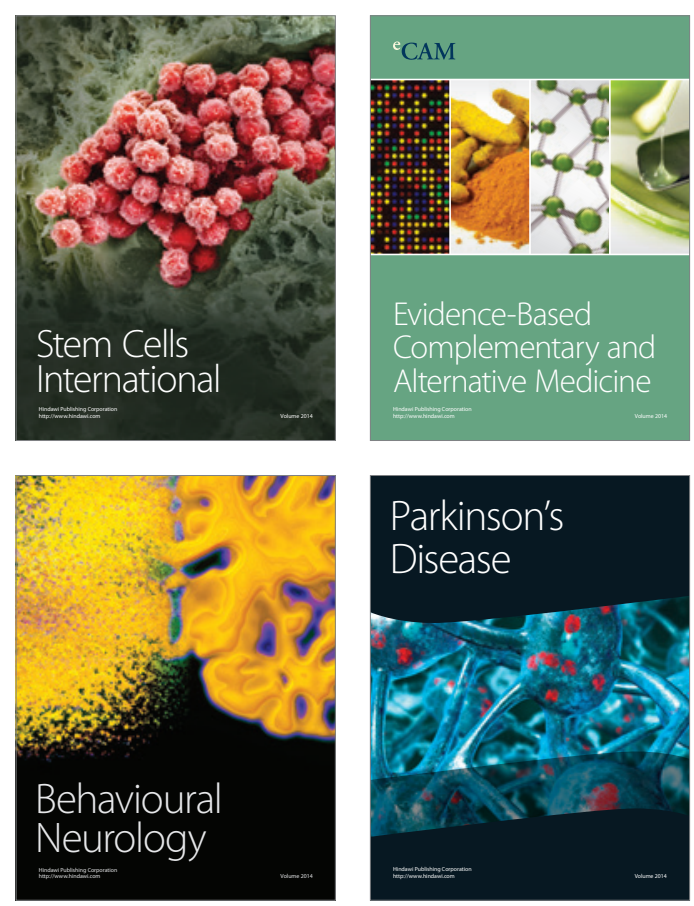

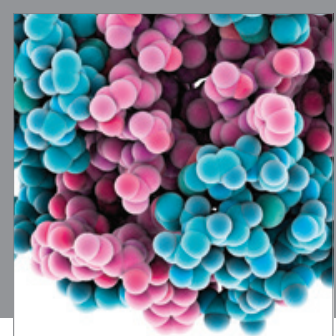

Journal of
Diabetes Research

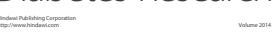

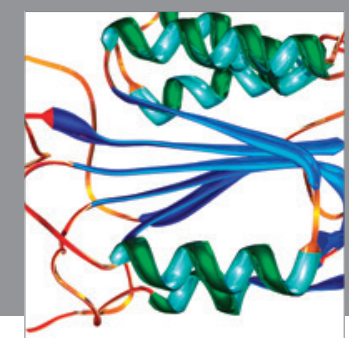

Disease Markers
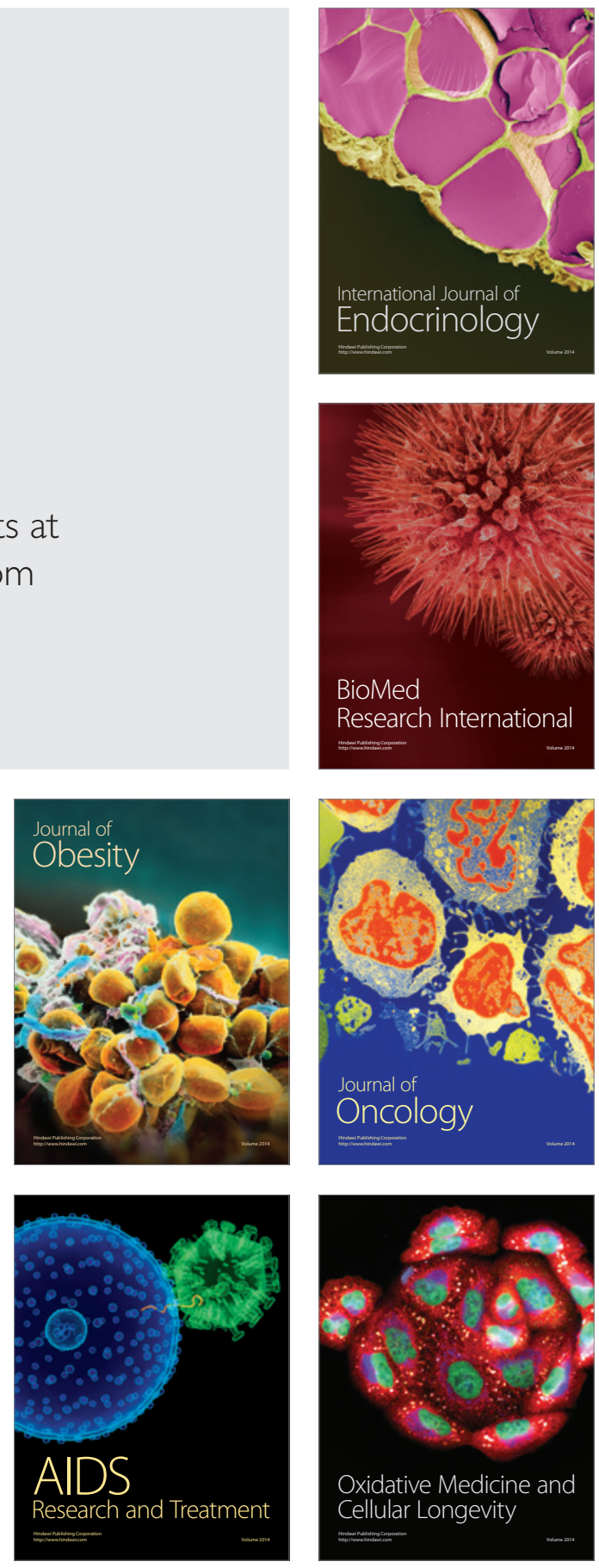